Documenta Ophthalmologica 97: 341-347, 1999.

T.J. Wolfensberger (ed.), Conference Proceedings of the $2^{\text {nd }}$ International

Symposium on Macular Edema, 137-143.

(C) 2000 Kluwer Academic Publishers. Printed in the Netherlands.

\title{
Novel imaging techniques for diabetic macular edema
}

\author{
CONCEIÇÃO LOBO ${ }^{1}$, RUI BERNARDES ${ }^{2}$, J. R. FARIA DE ABREU ${ }^{1}$ and \\ JOSÉ G. CUNHA-VAZ ${ }^{1}$ \\ ${ }^{1}$ Center of Ophthalmology, University Hospital and Institute for Biomedical Research in \\ Light and Image, 3000 Coimbra, Portugal; ${ }^{2}$ Center of Ophthalmology, Institute for \\ Biomedical Research in Light and Image, 3000 Coimbra, Portugal
}

\begin{abstract}
Retinal edema should be defined as any increase of water of the retinal tissue resulting in an increase in its volume. It may be of cytotoxic or vasogenic origin. Development of vasogenic macular edema is dependent on a series of factors such as blood pressure, bloodretinal barrier permeability, retinal cell damage, retinal tissue osmotic pressure and retinal tissue compliance. Objective measurements of retinal thickness are now possible using the Retinal Thickness Analyser. Localised measurements of blood-retinal barrier permeability may also be obtained using the Retinal Leakage Analyser, a modified confocal scanning laser fluorometer, while obtaining simultaneously angiographic images of the choroid and retina. These new imaging techniques show that cytotoxic and vasogenic retinal edema may occur independently in the early stages of diabetic retinopathy. These findings offer new perpectives for designing novel therapeutic strategies.
\end{abstract}

Key words: diabetes, edema, imaging, retinal leakage analyser, retinal thickness analyser, retinopathy

\section{Introduction}

Diabetes is the leading cause of new cases of legal blindness among working age Americans. Insulin dependent diabetes mellitus (IDDM) or Type 1 carries a higher risk of ocular complications, but because there are many more patients with non-insulin dependent diabetes mellitus (Type 2), the latter group comprises a substantial proportion of patients with blinding sequelae. It has been estimated in the United States that there are almost 75,000 new cases of diabetic macular edema each year. These numbers are clearly on the rise, and it is expected that by the year 2010, $10 \%$ of the American population will suffer from diabetes, especially Type 2 [1].

\section{Basic concepts of retinal edema}

First, it is very important to define retinal edema. Retinal edema should clearly 
be defined as any increase of water of the retinal tissue resulting in an increase in its volume, i.e., thickness.

This increase in water content of the retinal tissue may be initially intracellular or extracellular. In the first case, also called cytotoxic edema, there is an alteration of the cellular ionic exchanges with an excess of $\mathrm{Na}^{+}$inside the cell. In the second case, also called vasogenic edema, there is a predominantly extracellular accumulation of fluid directly associated with an alteration of the Blood-Retinal Barrier (BRB). In this latter situation Starling law applies and any loss of equilibrium between hydrostatic, oncotic and osmotic pressure gradients across the BRB contribute to further water movements and edema formation.

In cytotoxic edema, there is initially not a true edema, only a redistribution of water from its normal extracellular location to the intracellular space, causing cell damage, more edema and later release of vasoactive substances which may induce vasogenic edema through alteration of the BRB.

The initial alteration in intracellular, or cytotoxic, edema may be a lack of $\mathrm{ATP}^{+}$with depolarization of the cell membranes, alteration of the cell ionic pumps with an increase of extracellular $\mathrm{K}^{+}$with glutamate release and an increase of intracellular $\mathrm{Ca}^{+}$[2]. It may also result from an excitatory release of glutamate or lactic acidosis, activating $\mathrm{Na}^{+} / \mathrm{H}^{+}$transport and accumulation of intracellular $\mathrm{Na}^{+}$.

Vasogenic edema results from a breakdown of the BRB with extracellular deposition of macromolecules. The primary defect is in the BRB and the accumulation of fluid is extracellular.

In this situation, the 'force' driving water across the capillary wall is the result of a hydrostatic pressure difference $\Delta \mathrm{P}$ and an effective osmotic pressure difference $\Delta \pi \sigma$ [3]. The equation regulating movements across the BRB is, therefore:

$$
(\text { driving force })=L p\left[\left(P_{\text {plasma }}-P_{\text {tissue }}\right)-\sigma\left(\pi_{\text {plasma }}-\pi_{\text {tissue }}\right)\right]
$$

where Lp is the hydraulic conductivity or membrane permeability of the BRB and $\sigma$, an osmotic reflection coefficient, $\mathrm{P}_{\text {plasma }}$, the blood pressure, $\mathrm{P}_{\text {tissue }}$, the retinal tissue pressure, $\pi_{\text {plasma }}$, blood osmotic pressure and $\pi_{\text {tissue }}$, the tissue osmotic pressure.

The loss of equilibrium between these pressure gradients is of importance only after alteration of the Blood-Retinal Barrier (BRB), contributing then to water movements.

An increase in $\Delta \mathrm{P}$, contributing to retinal edema, may be due to an increase in $\mathrm{P}_{\text {plasma }}$ or a decrease in $\mathrm{P}_{\text {tissue }}$ or both. An increase in $\mathrm{P}_{\text {plasma }}$ due to increased systemic blood pressure does contribute to retinal edema formation only after loss of autoregulation of retinal blood flow and alteration of the structural 
characteristics of the BRB. A decrease in $\mathrm{P}_{\text {tissue }}$ is an important component that has not been given sufficient attention. Any loss in the cohesiveness of the retinal tissue due to pathologies such as cyst formation, vitreous traction, or pulling at the inner limiting membrane will lead to a decrease in $\mathrm{P}_{\text {tissue }}$. A decrease in $\mathrm{P}_{\text {tissue }}$, or increased retinal tissue compliance may lead to fluid accumulation, edema formation, and an increase in retinal thickness.

A decrease in $\Delta \pi$, contributing to retinal edema, may occur due to increased protein accumulation in the retina after breakdown of the BRB. Exsudate formation and extravasation of proteins will draw more water into the retina. This is the main factor provoking a decrease in $\Delta \pi$, as a reduction in plasma osmolarity high enough to contribute to edema formation is an extremely rare event.

After a breakdown of the BRB the progression of retinal edema depends directly on the $\Delta \mathrm{P}$ and $\Delta \pi$ gradients. In these situations, tissue compliance becomes more important, influencing directly the rate of edema progression.

In summary: retinal edema may be, initially, exclusively intracellular or extracellular. In the first case, it occurs without breakdown of the BRB. In the second case, a breakdown of the BRB must be present.

\section{Clinical evaluation of retinal edema}

The clinical evaluation of macular edema has been characterized by its difficulty. Direct and indirect ophthalmoscopy may show only an alteration of the foveal reflexes. Stereoscopic fundus photography and slit-lamp microscopy play an important role demonstrating changes in retinal volume in the macular area but they are dependent on the observer experience and the results do not offer a reproducible measurement of the volume change. The Early Treatment Diabetic Retinopathy Study, defined that the following characteristics indicate "clinically significant macular edema":

(1) Thickening of the retina (as seen either by slit lamp biomicroscopy or by stereo fundus photography) at or within 500 microns of the center of the macula;

(2) Hard exsudates at or within 500 microns of the center of the macula, associated with the thickening of the adjacent retina (but not residual hard exsudates remaining after disappearance of retinal thickening); and

(3) A zone, or zones, of retinal thickening one disc area or larger size, any part of which is within one disc diameter of the center of the macula.

This definition was proposed to take into special consideration the involvement of the center of the macula and its relationship to visual loss. 


\section{Imaging of macular edema}

We have seen that in order to develop retinal edema an increase in retinal volume must be present. This increase in volume is represented by an increase in retinal thickness because of the anatomical architecture of the retina. Recently, a new technique was become available that measures objectively overall retinal thickness, the Retinal Thickness Analyser (RTA) [4].

We have defined retinal edema as any increase of water of the retinal tissue resulting in an increase in its thickness. Any increase in retinal thickness is, therefore, a direct measurement of retinal edema. It is of particular interest that there are now two instruments capable of measuring non-invasively the thickness of the retina, the Retinal Thickness Analyser and Optical Coherence Tomography (OCT). Both methods depend on clear media but allow for very precise measurement of minor changes in retinal thickness. The Retinal Thickness Analyser measures a slit-lamp like image of the retina, using the internal limiting membrane and the retinal pigment epithelium as references. It is associated with reliable positioning in the retina and has very good reproducibility. It is of particular value in situations of retinal edema, without marked disorganization of the retina. Optical Coherence Tomography gives a color-coded image of the optical density of the various cellular components of the retina and is of special interest in the presence of advanced structural changes in the retina like cyst formation, and to demonstrate vitreal traction and surface abnormalitites of the retina.

BRB breakdown has been detected by fluorescein angiography and measured by vitreous fluorometry. It is now possible, using the Retinal Leakage Analyser (RLA), a new instrument developed by our group, to perform localised measurements of the permeability of the BRB with simultaneous imaging of the retina [5]. Thus, quantitative maps of retinal thickness and quantitative maps of retinal fluorescein leakage, indicative of BRB permeability can be obtained simultaneously, making sure that they are from the same location in the retina. All these examinations and measurements are performed in a clinical environment.

We have now, therefore, the means to acquire more data on retinal edema. By measuring retinal thickness we characterize the location of the edema and are capable of following its evolution. Next, it is important to determine whether it is associated with breakdown of the BRB, i.e., whether it is vasogenic. The absence of a breakdown of the BRB would indicate a situation of increased thickness due to intracellular or cytotoxic edema.

Furthermore, in a situation of vasogenic edema, information about $\Delta \mathrm{P}$ and $\Delta \pi$ may be obtained using the OCT [6]. The presence of cysts and vitreal traction can be demonstrated very easily by this instrument, indicating loss of cohesiveness of the retinal tissue and demonstrating situations facilitating 


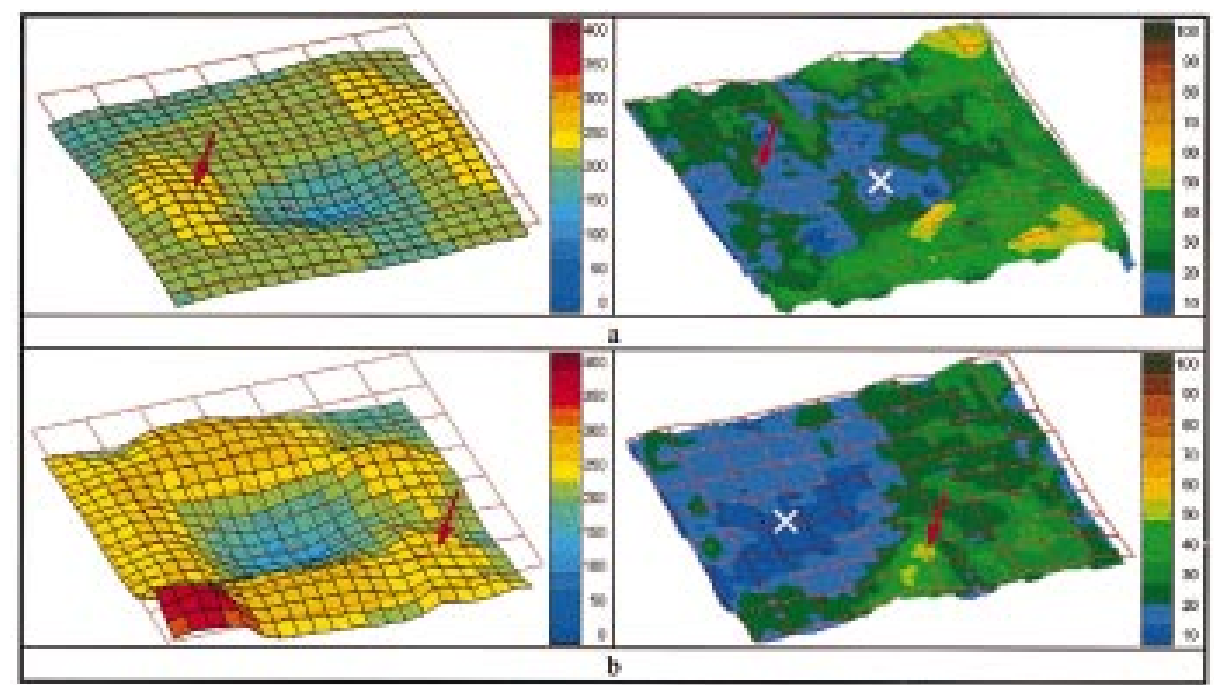

Figure 1. (a) Diabetic eye showing no visible signs of retinopathy on funduscopy. Left RTA - map of retinal thickness $(\mu \mathrm{m})$. Right - RLA - map of fluorescein leakage $\left(10^{-7} \mathrm{~cm} / \mathrm{s}\right)$ (normal values are in the range of blues and dark green; the white cross shows the center of the fovea). Note the presence of areas of thickening appearing independently from the leakage sites (arrows). (b). Diabetic eye with minimal retinopathy changes. Left - RTA - map of retinal thickness $(\mu \mathrm{m})$. Right - RLA - map of fluorescein leakage $\left(10^{-7} \mathrm{~cm} / \mathrm{s}\right)$ (normal values are in the range of blues and dark green; the white cross shows the center of the fovea). Note the zones of increased thickness correlated to sites of increased fluorescein leakage (arrows).

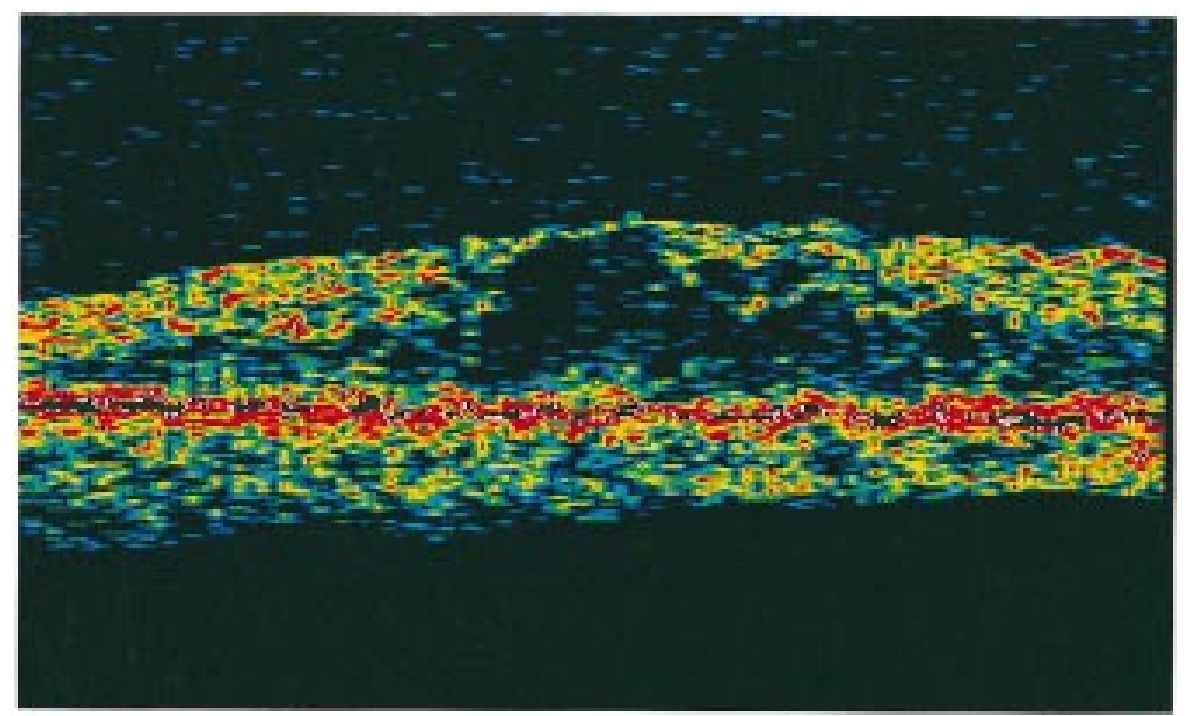

Figure 2. Image of Optical Coherence Tomography showing cyst formation in a situation of advanced diabetic macular edema. 
fluid accumulation in the retina. Accumulation of proteins and exsudates in the retina may also become apparent as a localised increase of optical density of the retinal structures.

With these new imaging methods it is now possible to clinically follow retinal edema and to obtain information about its type (cytotoxic or vasogenic), as well as about other important factors involved in its progression.

\section{Imaging of retinal edema in diabetes}

The application of these new methods to the evaluation of diabetic macular edema has shown that both types of retinal edema, cytotoxic and vasogenic, occur in the diabetic retina before the development of clinical significant macular edema.

Preliminary results have shown that in diabetic eyes without vascular pathology visible on ophthalmoscopic examination it is possible to find localized areas of increased retinal thickness without associated breakdown of the BRB in the posterior pole (Figure 1a). However, in other locations it is possible to find a clear association between zones of increased thickness and sites of increased fluorescein leakage and breakdown of the BRB (Figure 1b).

We think that in the diabetic retina the two types of retinal edema occur together from the initial steps of the disease. Cytotoxic edema may well be the first. Recent results from our laboratory indicate that the retinal vessels increase glucose transport into the retina in the presence of hyperglycemia, thus creating an excessive accumulation of glucose in the inner retina [7]. Abnormally elevated glucose levels in the retina in diabetes lead to an increase in lactate and frutose production. The increase in the ratio lactate-pyruvate is similar to that induced by hypoxia. Williamson and co-workers [8] have called this a situation 'pseudo hypoxia' induced by the hyperglycemia. A natural outcome is the development of intracellular ionic changes and excessive glutamate release causing intracellular edema. Later on, progressive retinal cellular damage would result in the release of vasoactive substances such as nitric oxide and free radicals, inducing vascular damage and breakdown of the BRB with protein leakage and extracellular edema (vasogenic).

Studies directed to the earliest stages of retinal involvement of diabetes using simultaneously the RTA and the RLA are already offering new insights into our understanding of diabetic retinal disease and diabetic retinal edema. In more advanced situations of retinal pathology associated with marked retinal edema, OCT examination may show alterations in retinal structure and cyst formation (Figure 2).

A better targeted therapy of diabetic retinal edema will be derived from the application of these new methods of retinal imaging. 
In diabetic macular edema, determining which type of retinal edema predominates (cytotoxic or vasogenic) may, in the future, influence the choice of neuroprotective or vasoprotective agents. Neuroprotective agents that are likely candidates include calcium-channel blockers, glutamate-receptor antagonists and antioxidants. Some of the vasoprotective agents that offer promising perspectives are nitric oxide synthethase inhibitors, Advanced Glycation Endproduct (AGE) formation inhibitors and Angiotensin Conversion Enzyme (ACE) inhibitors [9-11].

These drugs may be used in the near future to protect the retinal cells and the BRB from the damage caused by the increased and abnormal glucose metabolism occurring in the diabetic retina and thus delay the development of the retinopathy.

\section{References}

1. King H. The epidemic of NIDDM: an epidemiological prespective. Int Diab Fed Bull 1995; 40: 10-12.

2. Cohadon F. Protection Cérébrale. Bases Conceptuelles et Applications. Paris: Arnette Blackwell, 1995; 1-184.

3. Cunha-Vaz JG, Travassos A. Breakdown of the Blood-Retinal Barriers and Cystoid Macular Edema. Surv Ophthalmol 1984; 28 (suppl): 465-92.

4. Zeimer R, Mori MT, Khoobehi B. Feasibility test of a new method to measure retinal thickness noninvasively. Invest Ophthalmol Vis Sci 1989; 30: 2099-105.

5. Lobo C, Isidoro I, Simões PC, Leite E, Sander B, Cunha-Vaz JG. Topographic vitreous fluorometry using a modifed confocal laser scanning ophthalmoscope. Invest Ophthalmol Vis Sci 1996; 37 (suppl.): 611.

6. Puliafito CA, Hee MR, Lin CP, et al. Imaging of macular disease with optical coherence tomography. Ophthalmology 1995; 102: 217-29.

7. Murta JN, Serra, Cunha-Vaz JG. Characterization of D-glucose transport across diabetic retinal vessels. Invest Ophthalmol Vis Sci 1996; 37 (suppl.): 979.

8. van Enden M, Nyengaard J, Ostrair E, Burgan JH, Williamson JR. Elevated glucose levels increase retinal glycolysis and sorbitol pathway mechanisms. Invest Ophthalmol Vis Sci 1995; 36: 1675-85.

9. Corbett JÁ, Tilton RG, Chang K, et al. Aminoguanidine, a novel inhibitor of nitric oxide formation, prevents diabetic vascular dysfunction. Diabetes 1992; 41: 552-6.

10. Parving H, Larsen M, Hossonel E et al. Effect of antihypertensive treatment on bloodretinal barrier permeability to fluorescein in hypertensive type 1 (insulin-dependent) diabetic patients with background retinopathy. Diabetologia 1989; 32: 441-4.

11. Cunha-Vaz JG, Lobo C. Medical therapy of diabetic retinopathy. Exp Ophthalmol 1998; 24: $1-5$.

Address for correspondence: C. L. Lobo, Center of Ophthalmology, University Hospital and Institute for Biomedical Research in Light and Image, 3000 Coimbra, Portugal

Phone: 351-239-701182; Fax: 351-239-826665; E-mail: clobo@imagem.ibili.uc.pt 